\section{JTI}

JOURNAL OF

TRAUMA AND INJURY

\title{
Traumatic Separation of Bipartite Patella Underlying Gout
}

\author{
Eun Seok Choi, M.D., Ph.D. ${ }^{1}$ Jae Ang Sim, M.D., Ph.D. ${ }^{2}$, Jae Yun Go, M.D. ${ }^{2}$, \\ Young Gon Na, M.D. ${ }^{2}$ \\ ${ }^{1}$ Department of Orthopedic Surgery, Chungnam National University Hospital, Daejeon, Korea \\ ${ }^{2}$ Department of Orthopedic Surgery, Gachon University Gil Medical Center, Incheon, Korea
}

Received: July 23, 2018

Revised: October 9, 2018

Accepted: October 14, 2018 \begin{abstract}
la is a rare condition. This report presented a traumatic separation of bipartite patellar fragment caused by mild trauma in a patient with comorbid gout. When a patient with bipartite patella and underlying gouty arthritis complains of pain after trauma, clinical suspicion is needed about fragment separation of the bipartite patella.
\end{abstract}

Gouty arthritis is a common crystal arthropathy, but gout tophus in the bipartite patel-

Keywords: Patella; Gout; Injuries

\section{INTRODUCTION}

\section{Correspondence to}

Young Gon Na, M.D.

Department of Orthopedic Surgery, Gachon University Gil Medical Center,

21 Namdong-daero 774beon-gil,

Namdong-gu, Incheon 21565, Korea

Tel: $+82-32-460-3384$

Fax: $+82-32-423-3384$

E-mail: orthonyg@gmail.com
Gouty arthritis is a common crystal arthropathy caused by chronic hyperuricemia, resulting in monosodium urate crystal deposition around joints and tendons, but gout tophus in the bipartite patella is a rare condition [1-5]. Bipartite patella is mostly asymptomatic and is often conservatively treated even when symptomatic. However, when the fragment is separated, motion may cause pain and surgical treatment may be necessary [6]. For patients who present with patellar involvement of gout, the tophus sometimes induces a pathological fracture or joint malalignment since deposition of crystals in joints and bones leads to arthritis and bone destruction [1]. This article reports a case of gout tophus in the bipartite patella of 28-year-old man who had traumatic patellar separation. Informed consent was taken from the patient for using the medical records for the publication.

\section{CASE REPORT}

A 28-year-old male patient fell from a truck while loading stocks 4 months prior to 

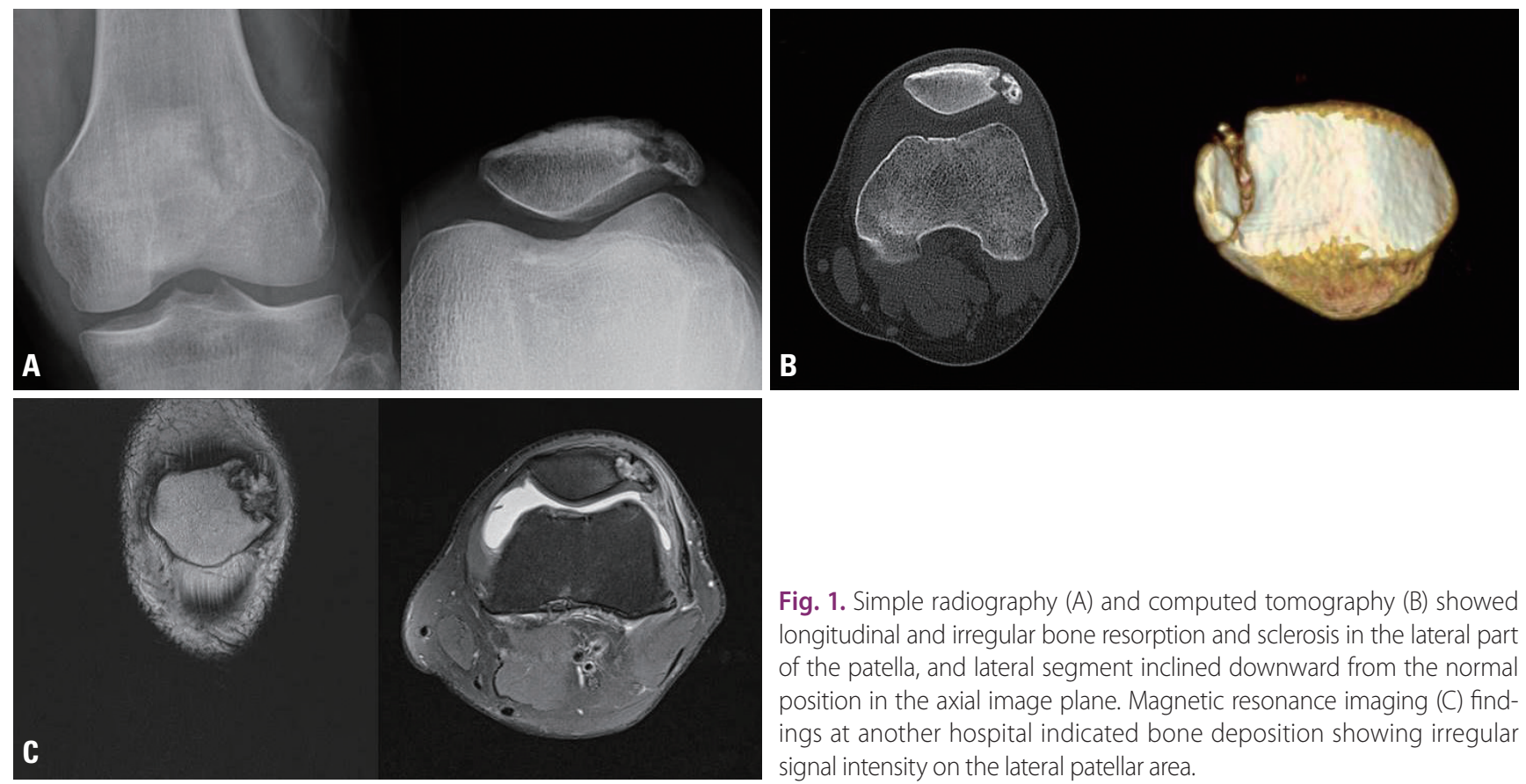

Fig. 1. Simple radiography (A) and computed tomography (B) showed longitudinal and irregular bone resorption and sclerosis in the lateral part of the patella, and lateral segment inclined downward from the normal position in the axial image plane. Magnetic resonance imaging (C) findings at another hospital indicated bone deposition showing irregular signal intensity on the lateral patellar area.

presenting at our clinic and struck his knee on the floor. The patient underwent conservative treatment with plaster cast fixation with a diagnosis of patellar fracture at another hospital. Despite 3 months of conservative treatment, the patient complained of persistent lateralsided pain upon flexion of the knee. He was transferred to our institution with a diagnosis of nonunion of the patella. The patient had no specific family history. $\mathrm{He}$ had a history of intermittent medication as needed for gout attacks. Laboratory findings were not specific other than $9.1 \mathrm{mg} / \mathrm{dL}$ of serum uric acid (normal range, 2.58.3), $48 \mathrm{~mm} / \mathrm{hr}$ of erythrocyte sedimentation rate (ESR; normal range, $0-10$ ) and $5.4 \mathrm{mg} / \mathrm{dL}$ of C-reactive protein (CRP, normal range; $0.0-0.5$ ). In the joint fluid analysis, the white blood cell (WBC) count was $23,696 / \mu \mathrm{L}$ with $87 \%$ of segmented neutrophil, and monosodium urate crystal was confirmed under polarized light microscopy. No organism was cultured from the joint fluid. There was no limitation of joint motion in physical examination, but there was pain on the anterior knee joint area upon flexion and on the lateral patella area upon compression. Simple radiography and computed tomography showed longitudinal and irregular bone resorption and sclerosis in the lateral part of the patella. The lateral segment was inclined downward from the normal position in the axial image plane. Magnetic resonance imaging (MRI) findings at the other hospital indicated bone deposition showing irregular signal intensity on the lateral patellar area (Fig. 1). As the patient came to our institution with a copy of the previous imaging studies, we could check the status of his left knee 3 years ago. The plain radiographs and the MRI showed typical findings of the bipartite patella only in the left knee (Fig. 2).

The patient's history, symptoms, physical examination, and radiological examinations indicated that his bipartite patellar fracture from the mild trauma may have been due to accelerated bone resorption and osseointegration caused by gout crystals deposited on the cartilage area of the patella. His symptoms were similar to those of lateral patella compression syndrome (LPCS) and were an indication for surgical treatment $[7,8]$.

There was no disruption of the periosteum on the operative findings. The fracture area was first examined using an image amplifier, and dissecting the periosteum showed yellowish brown joint fluid and grayish white precipitate (Fig. 3). The periosteum separation was done smoothly without additional maneuver and the separated cartilage margin was clean, while grayish white precipitate was de- 

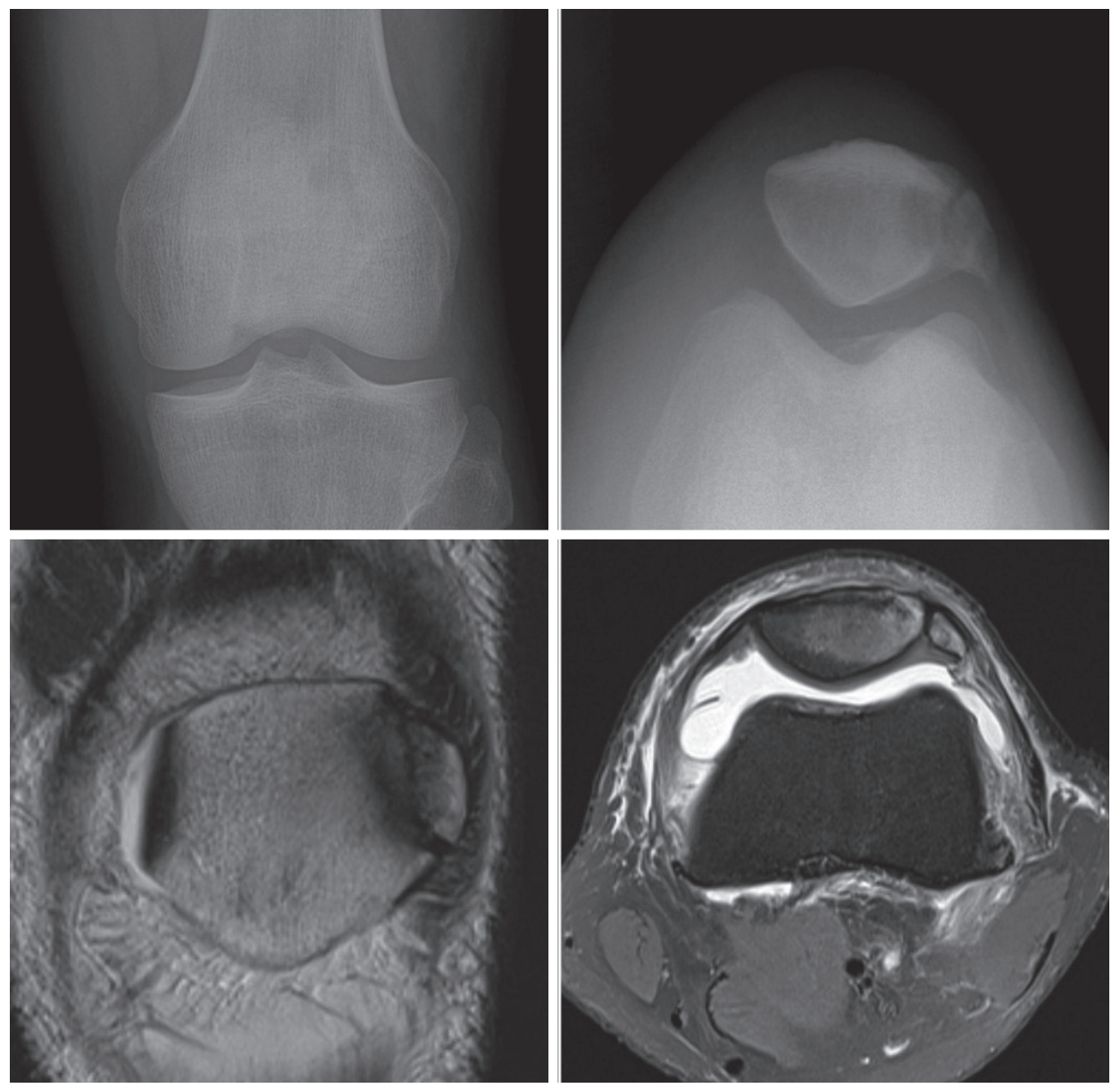

Fig. 2. Simple radiograph and magnetic resonance imaging which was taken 3 years earlier at other hospital. The images show typical finding of bipartite patella on his left knee.

posited on the bone site (Fig. 3). The surgical procedure followed the LPCS surgical procedures [7,8]. A small lateral patellar fragment causing LPCS-like symptom was removed first, leaving only the cortical bone attached to the lateral retinaculum for bony healing (Fig. 4). Several transverse tunnels were created in the main patellar fragment to pass the FiberWire ${ }^{\circledR}$ (Arthrex, Naples, FL, USA) sutures, and the lateral retinaculum was repaired using FiberWire ${ }^{\circledR}$ sutures. After surgery, hyperuricemia was managed with a urate-lowering agent, along with the analgesics. During rehabilitation, unprotected ambulation was encouraged immediately after the surgery while the range of motion was limited from 0 to 90 degrees for 4 weeks postoperatively. All exercises and daily activities were allowed after that. Two months after surgery, the patient returned to his pre-injury occupation, one that requires hard physical labor. One and half years after surgery, at the final exam, the patient had no pain and discomfort on his knee and returned to sports activity with 95 points of Lysholm score. On the simple radiograph at his final visit 18 months after surgery, lateral separate bony fragments were removed, and no patellar lateral subluxation was observed (Fig. 4).

\section{DISCUSSION}

Reports of bipartite patella prevalence in adults range from $0.2 \%$ to $6 \%$, but clinically significant symptoms occur only in approximately $2 \%$ of patients $[6,9]$. Painful bipartite patella is first treated conservatively, but when a patient does not respond to conservative treatment, the condition may be treated surgically [6]. While other etiologies may contribute, instability of the cartilage area of 


\section{JTI}
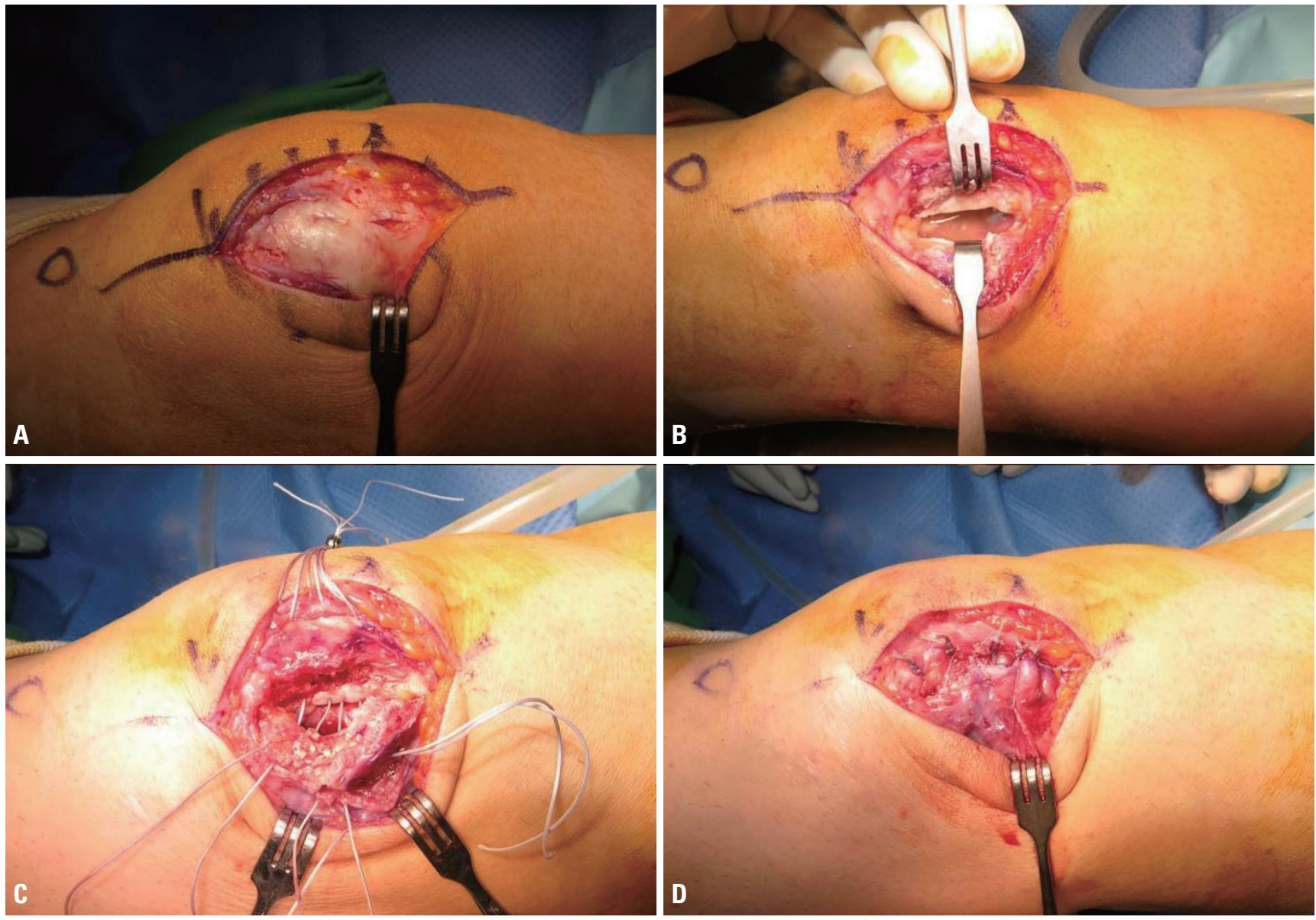

Fig. 3. (A) There was no disruption of the periosteum on the patella. (B) Once dissecting the periosteum, yellowish brown joint fluid and grayish white precipitate was visible between the main portion of the patella and bipartite patellar fragment. (C) Small lateral patellar bone that caused compression was removed first, and patellar tunnel was created for pull-out suture, leaving only the cortical bone attached to the lateral retinaculum, for bone to bone healing. (D) Lateral retinaculum was repaired by pull-out suture using 4 strands of FiberWire ${ }^{\oplus}$ (Arthrex, Naples, FL, USA).
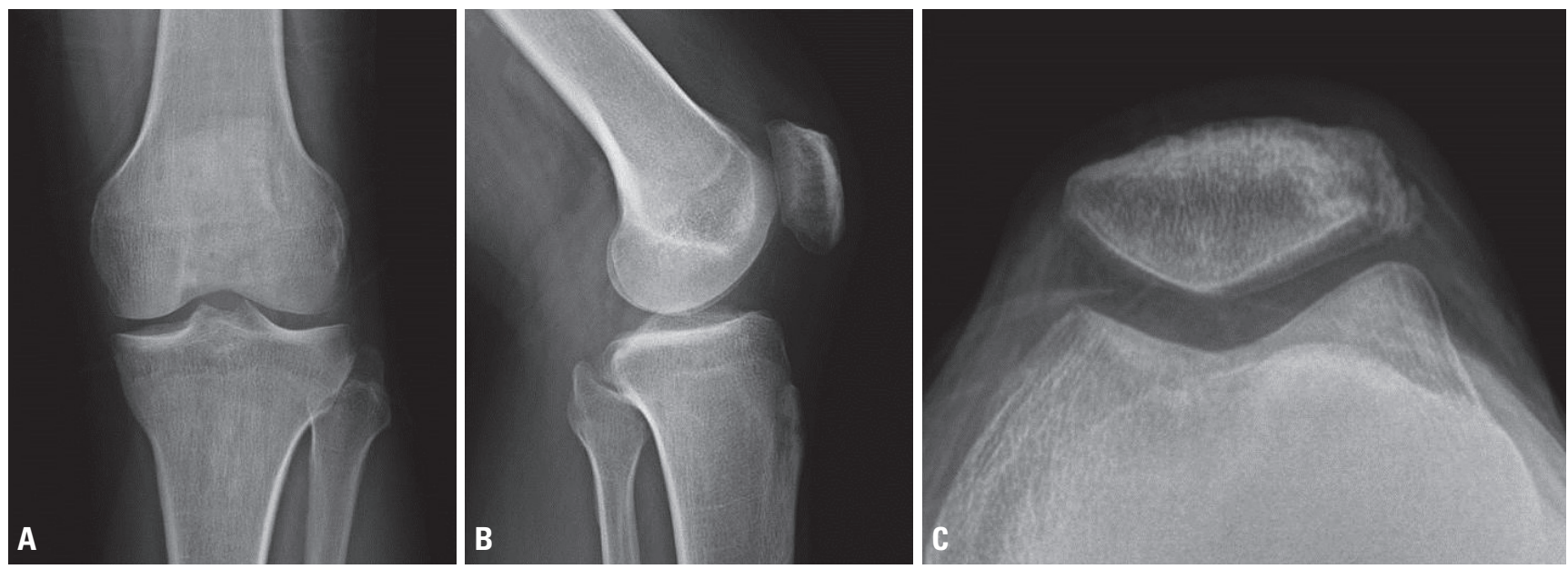

Fig. 4. Simple radiograph at 18 months after surgery: (A) anteroposterior view, (B) lateral view and (C) merchant view. Lateral bony fragment was excised, so no separate bony fragment was observed. 
a weak bipartite patella is believed to be the main cause of painful bipartite patella $[1,9,10]$. In this patient, separated bipartite patellar fragment might have caused persistent pain on the lateral aspect of the knee.

The patient's lateral aspect knee pain can also be explainable by the 'lateral patellar compression syndrome'. The lateral patellar compression syndrome causes pain due to increased abnormal contact pressure in the lateral patellofemoral joint in the flexion position. The management for this syndrome can be done by conservative or surgical treatment such as lateral retinaculum release [8]. In this case, separated bipartite patellar fragment might have elevated the contract pressure on the lateral aspect of the patellofemoral joint. As the lateral fragment of the patella was not large and was separated, we performed partial facetectomy, rather than re-fixation of the fragment [7].

Uric acid deposition of the bipartite patella is rarely seen in patients with gout, but several cases were reported in the literature [1-5]. These uric acid deposition is located on the cartilaginous part of the bipartite patella and on the surrounding bone, with erosion producing instability, which alone can lead to pain [1]. This patient is a rare case that the symptoms similar to those of lateral patellar compression syndrome, which produced by separation of the bipartite patella due to direct impact to the patella, with the pre-existing uric acid deposition in the bipartite patella.

This report presented a traumatic separation of bipartite patellar fragment caused by mild trauma in a patient with comorbid gout. Bipartite patella is a common congenital anomaly and is usually treated conservatively or may remain undiagnosed if asymptomatic. However, surgical treatment may be required if there is motion of the fragment, or if the fragment is large and causes symptoms such as lateral patellar compression syndrome, and does not respond to conservative treatment. Aggravation of underlying gout can contribute to the separation of the bipartite patellar fragment. Therefore, when a patient with bipartite patella and underlying gouty arthritis complains of pain after trauma, clinical suspicion is needed about fragment separation of the bipartite patella.

\section{ACKNOWLEDGEMENTS}

This study was performed at Gachon University Gil Medical Center.

\section{REFERENCES}

1. Enomoto H, Nagosi N, Okada E, Ota N, Iwabu S, Kamiishi S. Hemilaterally symptomatic bipartite patella associated with bone erosions arising from a gouty tophus: a case report. Knee 2006;13:474-7.

2. Hopper G, Gupta S, Bethapudi S, Ritchie D, Macduff E, Mahendra A. Tophaceous gout of the patella: a report of two cases. Case Rep Rheumatol 2012;2012:253693.

3. Kobayashi K, Deie M, Okuhara A, Adachi N, Yasumoto M, Ochi M. Tophaceous gout in the bipartite patella with intra-osseous and intra-articular lesions: a case report. J Orthop Surg (Hong Kong) 2005;13:199-202.

4. Reber P, Crevoisier X, Noesberger B. Unusual localisation of tophaceous gout. A report of four cases and review of the literature. Arch Orthop Trauma Surg 1996;115:297-9.

5. Tashiro S, Sugita T, Nakamura S, Kurata Y. Gout tophus in the bipartite patella. Orthopedics 2002;25:1295-6.

6. Atesok K, Doral MN, Lowe J, Finsterbush A. Symptomatic bipartite patella: treatment alternatives. J Am Acad Orthop Surg 2008;16:455-61.

7. Paulos LE, O'Connor DL, Karistinos A. Partial lateral patellar facetectomy for treatment of arthritis due to lateral patellar compression syndrome. Arthroscopy 2008;24:547-53.

8. Saper MG, Shneider DA. Diagnosis and treatment of lateral patellar compression syndrome. Arthrosc Tech 2014;3:e633-8.

9. Weckström M, Parviainen M, Pihlajamäki HK. Excision of painful bipartite patella: good long-term outcome in young adults. Clin Orthop Relat Res 2008;466:2848-55.

10. McMahon SE, LeRoux JA, Smith TO, Hing CB. The management of the painful bipartite patella: a systematic review. Knee Surg Sports Traumatol Arthrosc 2016;24:2798-805. 